Artículos Científicos - OPC - OBSERVACAFÉ - OURBE - OBSERVES

\title{
Construyendo indicadores locales de los ODS a través de los Observatorios Académicos de la Universidad Veracruzana
}

\section{Building local SDGs indicators through Academic Observatories at Universidad Veracruzana}

María de Lourdes Becerra Zavalaa_Edith Yolanda Romero Hernández ${ }^{b}$-María J. Luna Monzalvo $\_$-Blanca Inés Aguilar Frías ${ }^{d}-$ José Luis Pérez Chacón ${ }^{e}-$ Alberto García Leyva ${ }^{f}$

a Responsable del Observatorio de Políticas Culturales, Universidad Veracruzana, Xalapa, México.

Contacto: Ibecerra@uv.mx

${ }^{\mathrm{b}}$ Facultad de Nutrición, Universidad Veracruzana.

Xalapa, México. Contacto: edromero@uv.mx

c Miembro del Observatorio de la Cafeticultura Veracruzana, Universidad Veracruzana, Xalapa, México.

Contacto: jluna@uv.mx

d Responsable del Observatorio Urbano y de Arquitectura Sustentable, Universidad Veracruzana, Xalapa, México. Contacto: baguilar@uv.mx

e Universidad Veracruzana, Xalapa, México. Contacto: luperez@uv.mx

${ }^{f}$ Responsable del Observatorio de Economía y Sociedad, Universidad Veracruzana, Xalapa, México.

Contacto: albergarcia@uv.mx

Recibido: 31 de julio de 2020

Aceptado: 30 de septiembre de 2020

RESUMEN: Los Observatorios Académicos de la Universidad Veracruzana (UV) procesan y analizan información para explicar o aportar conceptos de una determinada realidad, mediante la identificación y propuesta de indicadores para el monitoreo de las variadas condiciones del hábitat Veracruzano. Frente a tales condiciones, se construyen espacios 


\section{Artículos Científicos - OPC - OBSERVACAFÉ - OURBE - OBSERVES}

sociales donde se genera conocimiento que fortalezca la responsabilidad social de la UV, acorde a la misión de la Coordinación Universitaria de Observatorios (CUO). En este contexto se desarrolló el foro alusivo a los Objetivos del Desarrollo Sostenible (ODS) establecidos por la Organización de las Naciones Unidas (ONU). Se presentan los resultados que coadyuvan de manera interdisciplinaria en la construcción de una perspectiva estatal de los ODS. En ellos se plantea una mirada reflexiva desde la academia donde se valoran escalas y áreas de interés de los diferentes participantes. Eso se cristaliza en propuestas de indicadores a nivel local con variables transversales, mediante metodología interdisciplinaria.

Palabras clave: Objetivos del Desarrollo Sostenible; observatorios; indicadores.

ABSTRACT: The Universidad Veracruzana (UV) academic observatories process and analyze information to explain or contribute concepts of a given reality, by identifying and proposing indicators to help monitoring different conditions of Veracruz. Faced with this conditions, social spaces are built where knowledge is generated to strengthens social responsibility of the UV in accordance with the mission of the University Observatories Coordination (CUO). In this context, a forum referring to the Sustainable Development Goals (SDGs) established by the United Nations (UN) was established. Results that contribute to the construction of the SDGs with a state perspective and an interdisciplinary view are presented. These results propose a reflective look from the scholar perspective, where scales and areas of interest of the different participants are valued. This is crystallized in proposals of indicators at the local level with transversal variables, through interdisciplinary methodology.

Keywords: Sustainable Development Goals; Observatories; Indicators.

\section{Introducción}

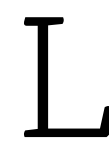

os crecientes desafíos mundiales: pobreza, hambre, desigualdad, paz y salud, han propiciado un llamado universal que les enfrente, proteja el planeta y garantice la paz, así como la prosperidad humana para 2030. Es así como, al adoptar la Agenda 2030 para el Desarrollo Sostenible, la Asamblea General de la Organización de las Naciones Unidas (ONU) y los estados firmantes conforman un plan de acción a favor de las personas, el planeta y la prosperidad; asimismo, reconocen que el mayor de estos desafíos es la erradicación de la pobreza y afirman que sin lograrlo no puede haber desarrollo sostenible.

Estamos resueltos a poner fin a la pobreza y el hambre en todo el mundo de aquí a 2030, a combatir las desigualdades dentro de los países y entre ellos, a construir sociedades pacíficas, justas e inclusivas, a proteger los derechos humanos y promover la igualdad 
Artículos Científicos - OPC - OBSERVACAFÉ - OURBE - OBSERVES

entre los géneros y el empoderamiento de las mujeres y las niñas, y a garantizar una protección duradera del planeta y sus recursos naturales (ONU, 2020).

Este compromiso común está organizado en 17 Objetivos de Desarrollo Sostenible (ODS) que regirán los programas de desarrollo mundial durante los próximos 15 años. Estos cuentan con 169 metas de carácter integrado e indivisible, en donde los Estados en plena soberanía fijan sus propias metas nacionales. Cabe destacar que, los ODS enfatizan

la erradicación del hambre y lograr la seguridad alimentaria; garantizar una vida sana y una educación de calidad; lograr la igualdad de género; asegurar el acceso al agua y la energía; promover el crecimiento económico sostenido; adoptar medidas urgentes contra el cambio climático; promover la paz y facilitar el acceso a la justicia (ONU, 2020).

\section{La Agenda 2030 en México y Veracruz}

Si bien la agenda 2030 inicia trabajos a principios de 2016, en México arranca el 26 de abril de 2017, cuando se publica en Diario Oficial de la Federación (DOF) el decreto por el que "se crea el Consejo Nacional de la Agenda 2030 como una instancia de vinculación del Ejecutivo Federal con los gobiernos locales, el sector privado, la sociedad civil y la academia" (Secretaría de Gobernación, 2017). Hacia enero de 2019 se relanza tal Consejo y queda presidido por la Oficina de la Presidencia de la República eintegrado por los titulares de las 18 Secretarías que conforman el gabinete presidencial, estructura que pretende una labor integral en torno a los ODS (Observatorio Regional de Planificación para el Desarrollo, \& de América Latina y el Caribe, 2020).

A nivel estatal, el gobierno del Veracruz en 2019 aprobó la creación del Consejo Veracruzano para el Desarrollo, integrado a su vez por tres comités técnicos: de Bienestar, de Derechos Humanos y de Economía, que permitan garantizar el cumplimiento de la Agenda 2030 para el Desarrollo Sostenible en el Estado de Veracruz. De manera tal que los comités se ocupen de coordinar, diseñar, ejecutar, monitorear y evaluar estrategias, programas, acciones y políticas públicas encaminadas al logro de los objetivos del desarrollo sostenible (Gobierno del estado de Veracruz, 2019). 


\section{La Universidad Veracruzana y los ODS}

Por su parte la Universidad Veracruzana (UV) señala en su visión al 2030, que contribuye al desarrollo sustentable con la vinculación efectiva con los sectores social y productivos, para la formación de ciudadanos éticos y competentes en el ámbito local y global (Universidad Veracruzana, 2019).

En este sentido, a fin de cumplir con el compromiso social que la distingue, de manera conjunta con la CUO, implementa acciones a través de los Observatorios Académicos que trabajan en 5 grandes áreas para contribuir al monitoreo de las condiciones socioambientales del estado, a la vez que procuran integrar los ODS en tres dimensiones del desarrollo sostenible (social, económico y ambiental) y que los agrupa de la siguiente manera:
A. Medio ambiente
B. Salud y bienestar
C. Desarrollo urbano
D. Aspectos sociales
E. Economía y política.

\section{Medio ambiente}

En la dimensión ambiental, se consideran los desafíos más importantes de los países de América Latina y del Caribe para lograr el desarrollo con un grado adecuado de sustentabilidad ambiental. Son evidentes las contradicciones que se dan ante las propuestas de desarrollo para la región, entre el crecimiento económico y el medio ambiente. Si bien, el problema es complejo se requiere una concepción integral del desarrollo, donde el medio ambiente sea un elemento inherente del mismo (Gligo, 2001).

Es necesario reconocer el carácter global de la crisis ambiental que afecta a todas las sociedades del planeta y que se denota una transformación más allá de lo ambiental: la estrecha relación entre los seres humanos en la producción de sus condiciones de vida y las relaciones establecidas con el conjunto del mundo natural, por ende, se demandan nuevas 
Artículos Científicos - OPC - OBSERVACAFÉ - OURBE - OBSERVES

formas de colaboración e interacción entre las ciencias humanas y las naturales. De manera tal que resalta que lo social y lo natural deben ser comprendidos en el marco más amplio de las interacciones entre los sistemas sociales y los sistemas naturales (Pérez-Verdi, 2011).

\section{Salud y Bienestar}

En materia de Salud y bienestar las acciones se enfocan al trabajo para contribuir al cumplimiento del Objetivo 3: Garantizar una vida sana y promover el bienestar para todos en todas las edades.

Salud y Bienestar significa garantizar una vida sana y promover el bienestar de todos a todas las edades y es muy importante para la construcción de sociedades prósperas. Epidemias como el VIH/SIDA medran donde el miedo y la discriminación limitan la capacidad de las personas para recibir los servicios que necesitan a fin de llevar una vida sana y productiva. Sin embargo, a pesar de los importantes avances que se han hecho en los últimos años en la mejora de la salud y el bienestar de las personas, todavía persisten desigualdades en el acceso a la asistencia sanitaria. Garantizar una vida sana para todos exige un compromiso firme, pero los beneficios compensan los costos. Las personas sanas son la base de las economías saludables. Se debe concienciar a nuestra comunidad sobre la importancia de la buena salud y de un estilo de vida saludable, y dar a conocer el derecho de todas las personas a acceder a servicios de salud de calidad (ONU, 2016).

\section{Desarrollo Urbano}

En materia de Desarrollo Urbano, las acciones deben contribuir a alcanzar el Objetivo 11: Ciudades y comunidades sostenibles que garanticen mejores condiciones de bienestar social y entornos saludables para toda la sociedad.

Más de la mitad de la población mundial vive hoy en zonas urbanas. En 2050, esa cifra habrá aumentado a 6.500 millones de personas, dos tercios de la humanidad. No es posible lograr un desarrollo sostenible sin transformar radicalmente la forma en que construimos y administramos los espacios urbanos. El rápido crecimiento de las urbes en el mundo en desarrollo -como resultado de la creciente población y del incremento en la migración- ha provocado un incremento explosivo de las mega urbes, especialmente en el mundo desarrollado, y los barrios marginales se están convirtiendo en una característica más significativa de la vida urbana (PNUD, 2020). 


\section{Artículos Científicos - OPC - OBSERVACAFÉ - OURBE - OBSERVES}

\section{Aspectos sociales}

La desigualdad es un asunto transversal en los ODS porque genera un espectro interdependiente de vulnerabilidad económica, salud, medio ambiente, violencia. En contraparte, un desarrollo social sostenible consideraría la inclusión social y económica, en relación a programas de sostenibilidad ambiental en el marco de paz y respeto de los derechos humanos (Presidencia de la República, AMEXID, y el Programa de Naciones Unidas para el Desarrollo, 2016). Las metas de los Objetivos 1, 8 y 10 se encaminan en ese mismo sentido. En la meta 4.7, se aboga por una educación que valore la diversidad cultural y promueva una cultura de paz y no violencia, así como por una contribución de la cultura al desarrollo sostenible. Otras metas como la 8.3 y 8.9 y 12b, refieren a la necesidad y promoción de actividades económicas relacionadas con el turismo sostenible, creatividad, innovación, y promoción de productos locales; al igual que la meta 11.4, que señala la necesidad de salvaguardar el patrimonio cultural y natural.

Promover el respeto y aprendizaje de la diversidad cultural en el marco de un enfoque basado en los derechos humanos propicia el entendimiento cultural y la paz, metas del que reclama sociedades pacíficas y justas e instituciones eficaces (...) La promoción de ese respeto también previene los conflictos y protege los derechos de los grupos marginados (Hosagrahar, 2017, p.14)

Esto pone de manifiesto la vinculación recíproca de los aspectos culturales con el resto de las dimensiones señaladas. La cultura también juega un papel relevante en las acciones que buscan abatir la desigualdad social. Las acciones en pro del desarrollo humano realizadas en los ámbitos de la salud y el bienestar y de la educación de calidad son interdependientes con el contexto cultural y las realidades comunitarias y ecológicas.

\section{Economía y política}

La Agenda 2030 plantea "promover el crecimiento económico sostenido, inclusivo y sostenible, el empleo pleno y productivo y el trabajo decente para todos." (Naciones Unidas, 2019, p.38).

El crecimiento económico sostenido e inclusivo puede impulsar el progreso, crear empleos decentes para todos y mejorar el nivel de vida. A nivel mundial, el PIB real per cápita y la productividad laboral han aumentado y el desempleo ha vuelto a caer a los niveles anteriores a la crisis financiera. Sin embargo, la 
Artículos Científicos - OPC - OBSERVACAFÉ - OURBE - OBSERVES

lentitud del crecimiento en general está generando un replanteamiento de las políticas económicas y sociales para alcanzar los objetivos de transformación del Objetivo 8: alcanzar las metas de crecimiento económico en los países menos adelantados; aumentar las oportunidades de empleo, en particular para los jóvenes; disminuir las desigualdades entre las regiones, los grupos de edad y los sexos; minimizar el empleo informal; y promover ambientes laborales seguros y protegidos para todos los trabajadores. (Naciones Unidas, 2019, p.38).

\title{
Método
}

Ante el creciente interés por coadyuvar con los fines de los ODS y en el marco del Plan General de Desarrollo 2030 de la UV, el cual señala en su Eje 4. Visión sistémica de temas transversales en la vida universitaria, que

\begin{abstract}
Los procesos derivados de las prácticas globales y las consecuencias de ellas implican nuevos retos para las IES. Los cuáles deben ser abordados desde diferentes perspectivas ...la sustentabilidad, la inclusión, la promoción de la salud, los derechos humanos y la justicia, el arte y la creatividad, son temas transversales que necesitan permear todas las funciones universitarias. Se deben integrar a partir de una visión sistémica de la propia universidad y del mundo en el sentido más amplio (Universidad Veracruzana, 2017:30)
\end{abstract}

La CUO organizó un Foro cuyo objetivo fue: "Establecer bases para un trabajo colaborativo que permita a los observatorios universitarios de la Universidad Veracruzana, generar datos e indicadores para formular una perspectiva estatal de los ODS" (CUO, 2020).

Para ello se convocó a participar a todo aquel que tuviese interés en la temática. En su registro debían indicar en cuál temática de los ODS se interesaban, si conocían los ODS, o bien colaboraban en algún proyecto relacionado. De esta manera, se contó con la participación de: Comunidad UV. Estudiantes y docentes-investigadores de Facultades y Centros de Investigación de todas las áreas académicas (artes, biológico-agropecuaria, ciencias de la salud, económico-administrativa, humanidades y técnica). Personal de entidades administrativas como Relaciones Internacionales, Coordinación Universitaria para la Sustentabilidad, Vinculación, Museo de Antropología, Estudios de Posgrado. Otras instituciones de educación superior. Instituto Tecnológico del Valle de Oaxaca, Universidad 


\section{Artículos Científicos - OPC - OBSERVACAFÉ - OURBE - OBSERVES}

Autónoma de Ciudad Juárez, Universidad Pedagógica Veracruzana. Dependencias gubernamentales estatales. Trabajadores o representantes de Secretarías de Gobierno, Medio Ambiente, Protección Civil, Salud, Seguridad Pública, Comisión Estatal de Derechos Humanos de Veracruz, el Consejo Estatal de Población (COESPO), el Sistema para el Desarrollo Integral de la Familia del Estado de Veracruz.

También estuvieron presentes algunas miembros de asociaciones civiles relacionadas con el medio ambiente de la ciudad de Xalapa. De este modo se reunieron miradas sobre contextos locales y formas de encarar las problemáticas que han sido delimitadas en los ODS, pero se han agudizado en las últimas décadas en la vida cotidiana de los veracruzanos.

Para construir una perspectiva de los ODS que revelara tanto las necesidades de información local como las fuentes de información disponibles, se organizaron 5 mesas de trabajo, cada una con líneas definidas:

1. Mesa Ambiental. Cambio climático y sus afectaciones en el Estado; agua: consumo y resiliencia; agrosistemas, agroindustria y explotación forestal; vida submarina y de ecosistemas del estado de Veracruz; lugares importantes para diversidad biológica y del agua dulce.

2. Mesa Salud y Bienestar. Seguridad alimentaria; salud infantil; investigación Médica.

3. Mesa Social. Educación inclusiva y de calidad; desigualdad social; violencia; violencia de género; cultura.

4. Mesa Económico-político. Acceso a la justicia para todos; instituciones eficaces e inclusivas que rindan cuentas; cooperación internacional en materia de ciencia y tecnología; situación migratoria; instituciones sólidas, justicia y paz; pobreza; gasto público; empleo.

5. Mesa Urbana. Producción de energías renovables; desarrollo rural; industria; infraestructura; comunidades y ciudades sostenibles.

A partir de la presentación del objetivo general del Foro y del panorama general de los ODS, se consideraron dos etapas. Primero se trató de sensibilizar a los participantes a través de la contextualización de lo que se realiza en México respecto a la medición de los ODS. Para ello, se contó con la participación de expertos en el tema que presentaron el panorama, desde una 
Artículos Científicos - OPC - OBSERVACAFÉ - OURBE - OBSERVES

mirada histórica al tema de la sostenibilidad; el Dr. Carlos Welsh Rodríguez presentó las rutas posibles de medición ante la inmensa cantidad de información generada al respecto. El Instituto Nacional de Estadística y Geografía (INEGI) mostró cómo y cuáles indicadores se han elaborado, así como todo lo que falta por construir para la medición regional y local de los ODS.

Posteriormente, ya establecidas las mesas de trabajo,_se procedió a la contextualización acorde a la temática correspondiente de cada una de estas, a partir de los objetivos y líneas previamente depurados por el equipo de trabajo de la CUO y Observatorios Académicos. Se privilegió el diálogo en plenaria, seleccionando los aspectos presentes, ausentes y necesarios a partir de las preguntas: ¿por qué y para qué medir localmente los ODs?; ¿cuáles son las metas e indicadores a las que se les puede dar seguimiento?; ¿quiénes podrían colaborar en un monitoreo de carácter local?; ¿qué otras formas de medirlos se podrían implementar?

Cada mesa identificó metas e indicadores específicos que deberían relacionarse con el entorno veracruzano. Se analizaron comparativa y críticamente las metas e indicadores de seguimiento a nivel nacional, al igual que los indicadores propuestos por el Consejo Veracruzano de la Agenda 2030. La jornada que duró aproximadamente nueve horas, concluyó con la plenaria de resultados por mesa, así como el esbozo de un plan de trabajo de observatorios universitarios liderado por la CUO.

Durante los meses posteriores al Foro, se colaboró en equipo dentro de lo que podría considerarse un procedimiento metodológico interdisciplinario compartido (Klein, 2010). Esto es, liderados por la CUO, se asumió una metodología de construcción de indicadores propia de la estadística: identificación de variables cuantitativas o cualitativas, conforme a las metas e información disponible.

Los resultados del Foro se sistematizaron en bitácoras orientadas por dos tópicos: cuál es el papel de los observatorios universitarios en cuanto al logro de los ODS en perspectiva estatal; y las condiciones existentes de información, así como de colaboración dentro y fuera de la UV. Posteriormente se analizaron en relación con manuales, informes, y propuestas de medición a nivel mundial y nacional. Se consideró pertinente dar seguimiento a la 
participación de los observatorios académicos UV en la medición de los ODS a mediano plazo mediante una encuesta para delimitar con mayor precisión cuales se medirán y a través de que metodologías e indicadores.

\section{Resultados}

Durante el trabajo de las mesas en el Foro se pudo observar que surgieron reflexiones comunes en todas las mesas: necesidad de un sistema de información común con acceso libre y oportuno para los interesados en el tema; el papel de los observatorios universitarios debería ser el de generar información de necesaria, de carácter local mediante indicadores y/o variables que reflejen las realidades locales; mantener la transversalidad de la información generada.

Lo anterior coincide con una visión holística que considera "valores, principios y estándares que regulan esquemas normativos internacionales y [...] objetivos verificables a corto plazo" (Presidencia de la República, AMEXID, y PNUD, 2016, p. 21). Esto es, se reconoce la necesidad de estándares comunes (ODS, información en común para la medición) conforme a las realidades locales para medir a corto y mediano plazo el logro de los ODS. En particular, la mesa Social planteó la necesidad de realizar diagnósticos para construir variables específicas

de indicadores locales, y transversalizar aspectos educativos con pobreza y vulnerabilidad social. La mesa Urbana señaló que la información generada debería contribuir estructurar mejor las políticas públicas. La mesa Economía y Política se mencionó que los indicadores existentes carecen de contexto local, por lo tanto, hay información poco relevante para saber si los ODS están implementándose en Veracruz.

Igualmente, se presentaron reflexiones en torno al concepto de desarrollo, visto no solo como asunto de necesidades y carencias, también de potencialidades y matices culturales que involucran valores humanos, basados en un enfoque incluyente de derechos humanos. Siguiendo el análisis sociocultural propuesto por Max-Neef (1994), los derechos humanos indican potencialidades humanas que requieren condiciones favorables para su fomento, y pueden convertirse en una agenda programática considerando las condiciones requeridas en las comunidades descritas como atributos existenciales (individuales y colectivos): libertad, 
Artículos Científicos - OPC - OBSERVACAFÉ - OURBE - OBSERVES

identidad, afecto, subsistencia imbricados en instituciones, símbolos, materialidad; realizados en lugares y tiempos específicos (Max-Neef, 1994).

En ese mismo sentido, la mesa Salud y Bienestar mencionó que, si no se trabaja con indicadores que midan salud en lugar de enfermedad, difícilmente podrán alcanzarse los objetivos propuestos. Coincidió con la mesa Social en la necesidad de indicadores cualitativos que revelen el bienestar y calidad de los programas gubernamentales: no es suficiente con que existan, por ejemplo, instituciones contra la violencia de género, es necesario tener información local sobre su impacto y prevención en el tema. Inclusión y derechos humanos son parte de la guía en la construcción de una mirada local que favorece la interdependencia de la información necesaria para pensar la sostenibilidad.

Los observatorios UV estarían buscando un camino propio en el siglo xxi en medio de crisis a escalas e impactos diferenciados (sanitarias, económicas, violencia), para problemas constituidos en procesos históricos que hoy vivimos. Por ejemplo, la diversidad entendida como heterogeneidad de formas de significar, representar, interactuar y comportarse, en un proceso de entendimiento mutuo con otro ajeno-extraño, y nos re-sitúa a nuestro propio marco de referencia (Rehaag, 2012); atravesada por procesos de exclusión o marginalidad que estructuran la desigualdad social. De ese Foro emergieron los principios de inclusión y bienestar humano que orientan este trabajo, y del cual se presentan los primeros resultados.

\section{Indicadores locales y Observatorios Académicos UV}

Los indicadores locales dan cuenta de las desigualdades, por lo que son relevantes para políticas públicas asentadas en la inclusión. Tienen un alto dominio de comunicación en el espacio público, contribuyen al seguimiento de proyectos y programas sociales y, por ende, al cumplimiento de metas acordadas en cumbres internacionales. Para estos indicadores locales se pueden discurrir cuatro elementos principales: responden a un modelo teórico, enlaza personas en comunicación, comparables, con capacidad de replicarse y sencillos, además de que reflejen la desigualdad de género (Phelan, 2008). 
En el cumplimiento de los ODS y la aportación de información pertinente, varios son los esfuerzos que se han realizado, como es el caso de Sánchez, García y Sisto (2018) quienes, mediante una investigación sobre ciudades sostenibles en España, mencionan que para depurar el proceso para el logro de los ODS a nivel municipal, se deben considerar las siguientes acciones: hacer uso de datos, enfocarse en desigualdades, originar el aprendizaje recíproco entre ciudades, abrir la colaboración a favor de cumplir con el gran reto del desarrollo sostenible, y por último el colaborar con el gobierno central.

Así, con las aportaciones sistematizadas en el Foro, se elaboraron algunos indicadores con variables transversales en por lo menos dos metas. Para su elaboración también se consideraron "las posibles aportaciones desde la docencia (...) política institucional y liderazgo social" (Alba Hidalgo, Benayas del Álamo, \& Blanco Portela, 2020, p. 12). A manera de ejemplo, en la siguiente tabla se presentan los indicadores para el Objetivo 4, que son transversales a los Objetivos 6. Agua limpia y saneamiento, 13. Lucha contra el cambio climático; 14. Flora y fauna acuáticas, y 15. Flora y fauna terrestres.

Tabla 1.

Indicadores para medir estatalmente el Objetivo ODS 4. Educación de calidad

\begin{tabular}{|c|c|}
\hline \multirow{3}{*}{4 inerentant } & Objetivo 4. Educación de calidad \\
\hline & Meta \\
\hline & Aplicación: Local y/o Municipal \\
\hline \multirow[t]{3}{*}{ Indicadores } & $\begin{array}{l}\text { Número de escuelas con implementación de tecnologías } \\
\text { sustentables (captación de agua, tratamiento de agua, } \\
\text { paneles solares) por nivel educativo }\end{array}$ \\
\hline & $\begin{array}{l}\text { Número de investigadores/académicos que realizan } \\
\text { actividades de divulgación social de la ciencia en medios } \\
\text { de comunicación masivos }\end{array}$ \\
\hline & $\begin{array}{l}\text { Número de publicaciones y/o artículos de divulgación } \\
\text { social de la ciencia que realizan en torno al tema ambiental } \\
\text { en los medios de comunicación masivos }\end{array}$ \\
\hline $\begin{array}{c}\text { Aportes a la docencia y la } \\
\text { investigación }\end{array}$ & $\begin{array}{l}\text { Medición del impacto que tienen los temas referentes a la } \\
\text { educación ambiental en los estudiantes y en sus } \\
\text { comunidades para que lleve a la prevención, } \\
\text { sensibilización y socialización del conocimiento. }\end{array}$ \\
\hline
\end{tabular}

Fuente: Coordinación Universitaria de Observatorios, 2020. 
Artículos Científicos - OPC - OBSERVACAFÉ - OURBE - OBSERVES

Es oportuno mencionar que, al momento de escribir este artículo se cuentan con alrededor de 40 indicadores que todavía deben evaluarse en sus condiciones básicas para ser aplicados por parte de los Observatorios Universitario UV. Se deberátrabajar en colaboración y coordinadamente con el Gobierno del Estado para no duplicar información, ya que los proveedores de datos para los Observatorios Académicos son todas las dependencias gubernamentales tanto estatales como federales, quienes a su vez tienen que informar a su área de competencia respectiva. Se considera que a través de la CUO se podrán analizar los datos para generar propuestas que sirvan de insumo para los tomadores de decisiones para la implementación de proyectos, programas o estrategias de intervención que incidan directamente en el logro de los objetivos y la metas de los ODS.

Como complemento y seguimiento al Foro realizado y gracias a la colaboración del personal de la CUO, se realizó una encuesta virtual, misma que fue enviada a los responsables de los observatorios académicos de la UV para qué contestaran las siguientes preguntas:

1.- ¿Ha considerado en la metodología del Observatorio del que es responsable, incorporar aspectos de los ODS?

Si la respuesta es afirmativa. ¿Cómo lo está planteando?

Si la respuesta es negativa. ¿Por qué?

2.- ¿Se ha considerado dentro del Observatorio, colaborar con otros observatorios para la medición transversal de los ODS a nivel estatal?

Si la respuesta es negativa. ¿Por qué?

Si la respuesta es afirmativa. ¿Cuáles observatorios?

3.- Como responsable de su Observatorio. ¿Qué propone para la medición de los ODS a nivel estatal? 


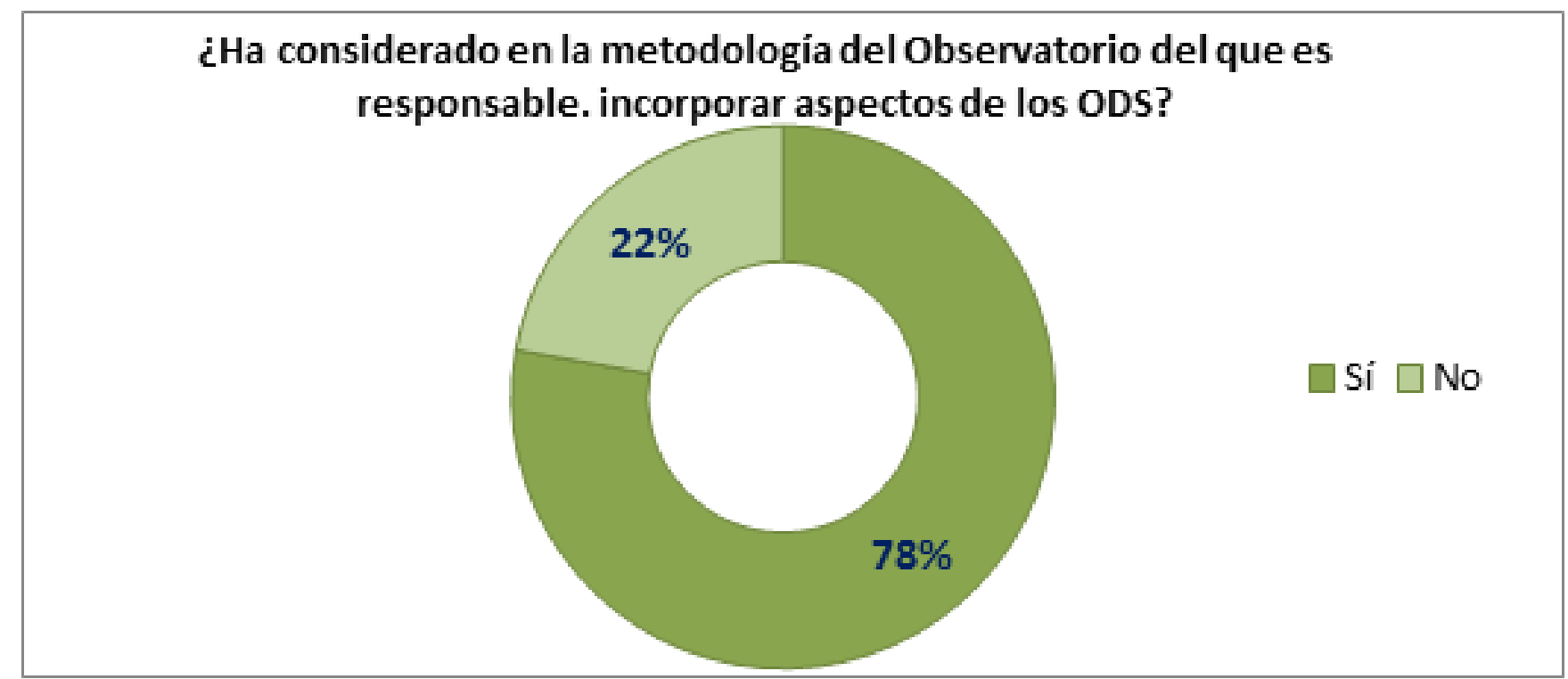

Figura 1. Respuestas cuantitativas de la encuesta virtual. Fuente: Elaboración propia a partir de información proporcionada por CUO y Observatorios UV

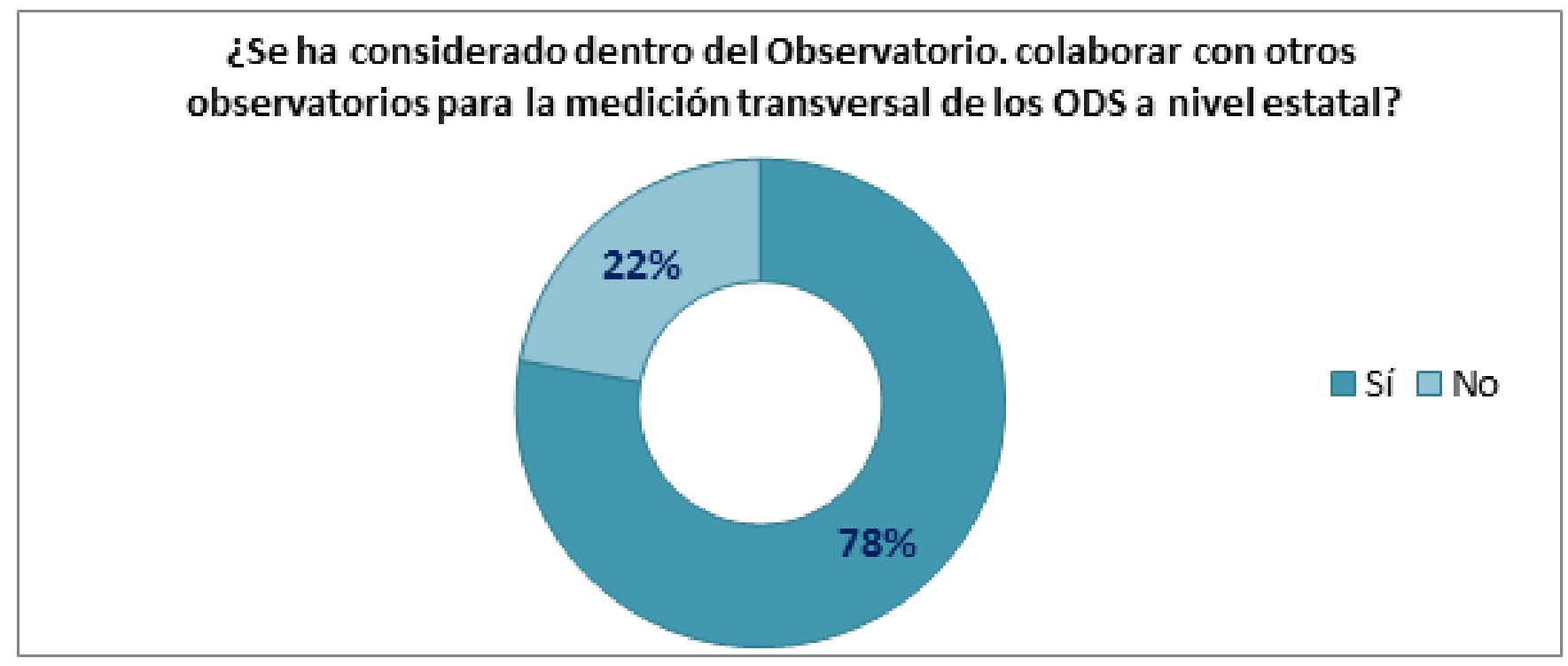

Figura 2. Respuestas cuantitativas de la encuesta virtual. Fuente: Elaboración propia a partir de información proporcionada por CUO y Observatorios UV

Los resultados fueron los siguientes: nueve de dieciocho observatorios colaboraron con sus respuestas. Como respuesta a la primera pregunta; ¿Ha considerado en la metodología del Observatorio del que es responsable, Incorporar aspectos de los ODS?, siete de los observatorios contestaron que síhan considerado en la metodología incorporar aspectos de los ODS y dos que no, de aquellos que contestaron la primera pregunta cómo afirmativa, la manera en la que lo están planteando es referente a objetivos específicos de los ODS, algunos 
en donde la temática del observatorio está enfocada al monitoreo de esos objetivos, otros aportando indicadores relacionados con los temas, otro observatorio como parte de la visión de la UV para contribuir para el 2025. Aquellos que contestaron negativa la pregunta, se refieren a que la metodología que emplean actualmente se adecúa para lo que miden, por lo cual, no pueden realizar la modificación a ODS, pero sí encuentran condiciones para colaborar con otros Observatorios Académicos UV. Además, que contestaron que no se remiten a temas específicos de los ODS pero que sí está relacionado de alguna manera con la temática que manejan.

Siete de los observatorios han considerado colaborar con otros para la medición transversal de los ODS, mientras que dos de ellos no lo consideran, ya que por una parte se contempla que el observatorio está en una fase inicial y la otra es por falta de tiempo, pero que sí han colaborado con otros observatorios para temas relacionados. Aquellos que contestaron afirmativa la pregunta; ¿Se ha considerado, dentro del Observatorio, colaborar con otros observatorios para la medición transversal de los ODS a nivel estatal?, han colaborado con por lo menos un observatorio o se tiene planeado colaborar, mientras que otros observatorios han participado hasta con hasta con 5.

Finalmente, y dando respuesta a una tercera pregunta; Como responsable de su Observatorio. ¿Qué propone para la medición de los ODS a nivel estatal?, las propuestas fueron variadas, desde aquellas que van desde una escala de medición, hasta metodologías híbridas que contemplan visores y matrices entre otras diversas técnicas, pasando por estudios transversales por una parte, y por otra indicadores interdisciplinarios, también se mencionó que la medición de los ODS fueran equiparables en las regiones en las que se mide y de seguir con la gestión de obtención de información con el sector público, así como el apoyo de la CUO para procurar los instrumentos de medición.

Existen así diferentes factores que se van agregando a la propuesta de la medición de los ODS de manera local, aunque también se deben tomar en cuenta los diferentes fenómenos que nacen a partir de este intento de mediciones, entre los que podemos mencionar el tema de la transparencia y los nuevos modelos de poder. Respecto a lo anterior Gilli (2017) menciona que la participación de la ciudadanía impulsada por las redes sociales y las 


\section{Artículos Científicos - OPC - OBSERVACAFÉ - OURBE - OBSERVES}

nacientes tecnologías refleja a la democracia y ha originado un cambio del poder global en los últimos años.

Por lo anterior, es indispensable tomar en cuenta la opinión de las personas, como quienes participaron en el Foro, a través de las nuevas herramientas que se tienen disponibles y que abonan a dar cuenta del avance a favor del cumplimiento de los ODS, ya que contribuye a cimentar la función de la sociedad, incluyendo academia y observatorios, y lo que es factible medir. Esto es también congruente con la política de responsabilidad social universitaria, pues el proceso deberá mantener la vinculación de manera colaborativa con diferentes sectores sociales en la generación y divulgación de la información, para la generación de propuestas de solución a través de la docencia/investigación.

\section{Conclusiones}

La Universidad Veracruzana, como Institución de Educación Superior (IES), construye relaciones con el entorno mediante su visión de respeto por el pensamiento local para articular su actuación a nivel global. La comunidad académica que la constituye proviene de la cultura del ser y del hacer de manera colaborativa desde cada disciplina. La generación de conocimientos y metodologías religadas a las prácticas, son características esenciales en sus tareas sustantivas. Docencia e investigación se entrelazan para dar pertinencia y relevancia a la formación profesional y humana. Estos principios están plasmados en su modelo educativo, que privilegia el aprendizaje centrado en el estudiante para lo largo de su vida.

Bajo esta premisa se celebró el Foro: “Los observatorios universitarios de la UV, construyendo una perspectiva estatal de los ODS". Este se constituyó en una experiencia académicaciudadana, que ha permitido a la UV apuntalar la importancia que implican los 17 ODS. De esta manera la universidad se suma a la agenda de desarrollo sostenible común entre los tres niveles de gobierno, el sector privado, la sociedad civil y las IES en nuestro estado. Como resultado del Foro, desde el ámbito académico, se privilegia la responsabilidad social y la capacidad de cooperación que cada actor asume en el cumplimiento de las metas propuestas para cada uno de los objetivos.

Los ODS fueron pensados de manera planetaria para atender la situación emergente que vive la humanidad, en sus múltiples interacciones ecosistémicas: desde lo social, lo cultural, lo 
Artículos Científicos - OPC - OBSERVACAFÉ - OURBE - OBSERVES

económico y lo político, esencialmente. Como política global del bienestar humano, pretende atender los efectos adversos en la salud, la educación, la cultura, y en general, en la vida en todas sus manifestaciones. Hemos llegado a un punto crucial, señala Fritjof Capra (1998), como consecuencia del paradigma mecanicista en el que se han fundamentado las interacciones humanas en el planeta. Así, los gobiernos buscan un bienestar de la humanidad a mediano plazo, ¿será esto posible dada la diversidad de formas de vida que cada comunidad ha logrado integrar como orden y control social?

Muchos son los esfuerzos que se han realizado en las diferentes partes del mundo, que se pueden traducir en la necesidad de constituir nuevos indicadores enfocados a plasmar las condiciones locales, en donde se vuelve importante la contribución de nuevas metodologías que expongan la evolución de los fenómenos que aquejan a la sociedad en ese nivel, y que contribuyen a la formulación y evaluación de las políticas públicas enfocadas a cumplir los ODS.

Estos objetivos suelen concebirse como un modelo a seguir de manera global, para que los países asuman las responsabilidades y sumen esfuerzos para su cumplimiento. Es la heterogeneidad de la sociedad la que obliga a una nueva manera de abordar la problemática y medir los fenómenos que la rodean, esta vez, desde las condiciones y circunstancias a partir de las necesidades propias de las localidades, regiones y/o ciudades. Es en este nivel, que diferentes actores de la sociedad aportan una serie de metodologías, ideas y conductos originarios en la problemática particular del contexto, que, desde la perspectiva propia de cada localidad, suman al esfuerzo global por su cumplimiento.

Representan el mayor reto de las Naciones Unidas y los estados miembros, en este transcurso, la academia y su cambio de paradigma, debe tomar en cuenta a los ODS para abonar al conocimiento, intencionalidad y discernimiento, por lo que cumplirá en ser una función clave en el cumplimiento de los objetivos (Cosme, 2018).

La adopción de los ODS, dentro de las políticas públicas para el bienestar social, implican en su conjunto, un análisis a conciencia sobre su cumplimiento en el orden social, sobre todo en los ámbitos local y regional. De ahí que se considere a la etnometodología (Baert y Carreira da 


\section{Artículos Científicos - OPC - OBSERVACAFÉ - OURBE - OBSERVES}

Silva 2011), como una alternativa de pensamiento epistémico para el estudio de caso y de buenas prácticas, que surgen en la cotidianidad del ciudadano común, de utilizar sus saberes tradicionales y populares para dar sentido a su cohesión social.

Tal es el caso de Veracruz, donde cohabitan innumerables comunidades, varias provenientes de un pasado precolombino como pueblos originarios, comunidades campesinas mestizas y ciudades medias que conforman áreas metropolitanas. Cada lugar o integración humana ha conseguido integrar su propia identidad sociocultural, basando sus relaciones intersubjetivas en procesos de intercambio de bienes, servicios y formas de vida, que no necesariamente obedecen a dinámicas (de movilidad socioeconómica) ajenas a lo local y lo comunitario.

Por ejemplo, en el área del Totonacapan, comprendida entre las cuencas del Río La Antigua y la cuenca del Río Cazones, viven los litutunaku (totonacos) quienes comparten territorio y prácticas socioculturales con nahuas, tepehuas, hñänñu (otomíes) y teenek (huastecos). La organización social de los totonacas se basa en el Consejo de los Abuelos, una práctica milenaria que se rescata en el Kantiyan del Centro de las Artes Indígenas, que ha sido reconocida en el año 2012 por la Organización de las Naciones Unidas para la Educación, la Ciencia y la Cultura (UNESCO) por su contribución a la salvaguardia del patrimonio cultural inmaterial. Los Abuelos indican que este reconocimiento se les hace porque en sus prácticas cotidianas de forma de vida se cumplen los 17 ODS.,

Por ende, se considera necesaria la etnometodología para plantear la alternativa de pensamiento epistémico, mediante el rescate oral, la entrevista y el trabajo de campo; para rescatar y recabar, de propia voz, las evidencias que muestren indicadores pertinentes y relevantes al cumplimiento de los ODS a nivel local y regional.

Desde este punto de vista, la contribución académica se vuelve fundamental, sobre todo en la conformación de nuevos indicadores. Rodríguez y López (2011) mencionan que hoy en día existe la asequibilidad económica de ajustar indicadores al análisis del territorio local, con indicadores basados en el desarrollo de potenciales, necesidades, y no en satisfactores. La incorporación de lo territorial permite abordar problemas específicos, y en este caso generar información que responda a situaciones donde se reconoce la heterogeneidad de actores sociales. Es en este proceso que los observatorios académicos llevan a la práctica la 
Artículos Científicos - OPC - OBSERVACAFÉ - OURBE - OBSERVES

vinculación con otros sectores sociales, a través de la docencia/investigación y el diálogo basado en el respeto y fomento a la diversidad.

\section{Agradecimientos}

A todo el equipo de la Coordinación Universitaria de Observatorios, liderado por el Mtro. José Ohtón Flores Consejo, por su invaluable apoyo en el proceso de organización, asesoría, recuperación, sistematización y análisis de información, que ha permitido la elaboración de este artículo.

\section{Referencias}

Alba Hidalgo, D., Benayas del Álamo, J., \& Blanco Portela, N. (2020). Cómo evaluar los ODS en las universidades. Red Española para el Desarrollo Sostenible. Recuperado de: https://reds-sdsn.es/wp-content/uploads/2020/04/Gui\%CC\%81a-COMO-EVALUARODS-2020-AAFF.pdf

Baert, P. y Carreira da Silva, F. (2011). La Teoría Social Contemporánea. En La Etnometodología (pp. 124-141). Madrid: Alianza Editorial.

Capra, F. (1998). El punto crucial, sociedad y cultura naciente. Buenos Aires: Troquel.

CUO. (2020, julio 9). Foro: "Los observatorios universitarios de la UV, construyendo una perspectiva estatal de los ODS"[Académica]. Coordinación Universitaria de Observatorios (CUO). Recuperado de: https://www.uv.mx/cuo/registro-ods/

Cosme, J. (2018). Los Objetivos de Desarrollo Sostenible y la academia. MEDISAN, 22(8), 838848. Recuperado de: https://www.redalyc.org/articulo.oa?id=368457320016

Gilli, J. J. (2017). La transparencia como objetivo del desarrollo sostenible. Ciencias

Administrativas, (9), 43-49. Recuperado de:

https://www.redalyc.org/articulo.oa?id=511653847004

Gligo, N. (2001). La dimensión ambiental en el desarrollo de América Latina. Santiago: CEPAL. Recuperado de: https://repositorio.cepal.org/handle/11362/2262

Gobierno del Estado de Veracruz. (2019). Plan Veracruzano de Desarrollo. Poder Ejecutivo y Oficina de Programa de Gobierno. Recuperado de: http://repositorio.veracruz.gob.mx/wp- 


\section{Artículos Científicos - OPC - OBSERVACAFÉ - OURBE - OBSERVES}

content/uploads/sites/4/files/transp/pvd_2019_2024/Gac2019-

224_Miercoles_05_TOMO_II_Ext_(PLAN_VERACRUZANO_2019_2024).pdf

Hosagrahar, J. (2017). La cultura, elemento central de los ODS. Correo de la UNESCO, 1, 12-14.

Recuperado de: https://es.unesco.org/courier/april-june-2017/cultura-elementocentral-ods

Klein, J. T. (2010). A taxonomy of interdisciplinarity. En R. Frodeman, J. T. Klein, \& C. Mitcham (Eds.), The Oxford handbook of Interdisciplinarity (First, pp. 15-30). New York: Oxford University Press.

Max-Neef, M. A. (1994). Desarrollo a escala humana: Conceptos, aplicaciones y algunas reflexiones (1. ed). Barcelona: Icaria.

Naciones Unidas. (2019). Informe de los Objetivos de Desarrollo Sostenible 2019. Nueva York: Naciones Unidas. Recuperado de: https://unstats.un.org/sdgs/report/2019/TheSustainable-Development-Goals-Report-2019_Spanish.pdf

Observatorio Regional de Planificación para el Desarrollo, \& de América Latina y el Caribe. (2020, julio 27). Consejo Nacional de la Agenda 2030 para el Desarrollo Sostenible De México. Observatorio Regional de Planificación para el Desarrollo [Académica]. Recuperado de: https://observatorioplanificacion.cepal.org/es/instituciones/consejonacional-de-la-agenda-2030-para-el-desarrollo-sostenible-de-mexico

ONU. (2016). Salud y bienestar. Por qué es importante. Recuperado de: https://www.un.org/sustainabledevelopment/es/wpcontent/uploads/sites/3/2016/10/3_Spanish_Why_it_Matters.pdf

ONU. (2020, julio 9). La Asamblea General adopta la Agenda 2030 para el Desarrollo Sostenible [Cooperación $\quad$ Internacional]. Desarrollo Sostenible. Recuperado de: https://www.un.org/sustainabledevelopment/es/2015/09/la-asamblea-generaladopta-la-agenda-2030-para-el-desarrollo-sostenible/

Pérez-Verdi, R. (2011). Ambientalismo y desarrollo sustentable: tramas del sistema capitalista. LiminaR, 9(2), 181-199.

Recuperado

de http://www.scielo.org.mx/scielo.php?script=sci_arttext\&pid=S1665$80272011000200012 \& \operatorname{lng}=e s \& t \operatorname{lng}=e s$.

Phelan, M. (2008). Una aproximación metodológica a los indicadores locales y comunitarios. Entre lo institucional y lo popular. Espacio Abierto, 173), 391-408. Recuperado de: https://www.redalyc.org/articulo.oa?id=12217302

PNUD. (2020, julio 1). Objetivo 11: Ciudades y comunidades sostenibles [Académica]. Objetivos de Desarrollo Sostenible. Recuperado de: 
Artículos Científicos - OPC - OBSERVACAFÉ - OURBE - OBSERVES

https://www.undp.org/content/undp/es/home/sustainable-development-goals/goal11-sustainable-cities-and-communities.html

Presidencia de la República, AMEXID, \& Programa de Naciones Unidas para el Desarrollo. (2016). Inclusión social: Marco teórico conceptual para la generación de indicadores asociados a los objetivos de desarrollo sostenible. Ciudad de México: PNUD. Recuperado de: http://www.mx.undp.org/content/mexico/es/home/library/poverty/inclusionsocial--marco-teorico-conceptual-para-la-generacion-de.html

Rehaag, I. (2012). Reflexiones acerca de la interculturalidad. CPU-e, Revista de Investigación Educativa, (2), 172-180. https://doi.org/10.25009/cpue.v0i2.162

Rodríguez, G., \& López, M. T. (2011). La importancia de la especificidad territorial en la construcción de indicadores locales. CIENCIA ergo-sum, Revista Científica Multidisciplinaria de Prospectiva, 18(2), 145-152. Recuperado de:

https://www.redalyc.org/articulo.oa?id=10418753005

Sánchez, I., García, J., Sisto, R. (2018). Los Objetivos de Desarrollo Sostenible en 100 ciudades españolas. Madrid: Red Española para el Desarrollo Sostenible (REDS). Recuperado de: https://reds-sdsn.es/wp-content/uploads/2018/10/Informe-ODS-en-100-ciudades2018-I-Resumen-web.pdf

Universidad Veracruzana (2017). Plan General de Desarrollo. Recuperado de: https://www.uv.mx/veracruz/odontologia/files/2017/07/UV-Plan-General-2030.pdf Universidad Veracruzana. (2019). Misión y visión [Académica]. https://www.uv.mx/universidad/mision-y-vision/ 